\title{
Internet-based analysis of availability of cardiopulmonary resuscitation courses for lay people in India, Nigeria and the United Kingdom
}

\section{Alexei Birkun, Fatima Trunkwala, Adhish Gautam, Miriam Okoroanyanwu, Adesokan Oyewumi}

Medical Academy named after S. I. Georgievsky of V. I. Vernadsky Crimean Federal University, Simferopol, Russian Federation; e-mail: birkunalexei@gmail.com

Purpose of the study. To analyze the availability and characteristic features of basic life support (BLS) courses offered to the general public in India, Nigeria and the UK.

Materials and methods. Google engine was utilized to perform the country-specific search of websites proposing BLS training courses for adult laypersons. The countries were selected based on the investigators' citizenship. The search was conducted in English on December 14, 2018. Geography of on-site training courses was limited to the country's territory. Data were analyzed descriptively.

Results. The search revealed 53, 29 and 208 BLS courses for India, Nigeria and the UK, respectively (see Table). The number of courses per 10 million inhabitants in India (0.4) and Nigeria (1.5) is, respectively, 79 and 21 times lower than in the UK (31.5). In India, the ratio is 3.7 times lower than in Nigeria. Geographical distribution of the courses is limited to $28 \%$ states and one union territory in India, 30\% states and the Federal Capital Territory in Nigeria. Within the UK, the on-site BLS training is offered in all constituent countries, with the highest prevalence in England (73\% of all UK's courses). BLS courses are mainly taught in a classroom ( $\geq 62 \%)$. The courses are highly variable in their duration (from 19 minutes to 9 days), group size (6-30 trainees), instructors' qualifications and participation cost. There are no free of charge on-site training proposals for laypersons in India or Nigeria (vs. $5 \%$ in the UK), and the mean cost of participation in these countries is generally above the monthly minimum wage.
Table. Characteristics of the BLS courses by country.

\begin{tabular}{|c|c|c|c|}
\hline Parameters & India & Nigeria & $\begin{array}{c}\text { United } \\
\text { Kingdom }\end{array}$ \\
\hline BLS courses, $n$ & 53 & 29 & 208 \\
\hline first aid courses, ${ }^{*}$ (\%) & $29(55)$ & $24(83)$ & $141(68)$ \\
\hline BLS-devoted courses, n (\%) & $24(45)$ & $5(17)$ & $67(32)$ \\
\hline Course organizers, $n$ & 33 & 20 & 95 \\
\hline non-profit organizations, $\mathrm{n}(\%)$ & $6(18)$ & $0(0)$ & $11(12)$ \\
\hline limited companies/corporations, $\mathrm{n}(\%)$ & $16(48)$ & $17(85)$ & $58(61)$ \\
\hline NS, $n$ (\%) & $11(33)$ & $3(15)$ & $26(27)$ \\
\hline \multicolumn{4}{|l|}{ Learning method $^{+}$} \\
\hline classroom, $\mathrm{n}(\%)$ & $33(62)$ & $22(71)$ & $191(92)$ \\
\hline distance, $\mathrm{n}(\%)$ & $20(38)$ & $4(13)$ & $7(3)$ \\
\hline blended, $\mathrm{n}(\%)$ & $0(0)$ & $3(10)$ & $10(5)$ \\
\hline NS, $n$ (\%) & $0(0)$ & $2(6)$ & $0(0)$ \\
\hline AED included, $\mathrm{n}(\%)$ & $31(58)$ & $15(52)$ & $112(54)$ \\
\hline Knowledge/skills assessment included, n (\%) & $26(49)$ & $18(62)$ & $114(55)$ \\
\hline Certificate awarded, n (\%) & $39(74)$ & $24(83)$ & $161(77)$ \\
\hline \multicolumn{4}{|l|}{ Course duration } \\
\hline first aid courses (classroom/blended), range & $\begin{array}{l}3 \text { hours - } \\
9 \text { days }\end{array}$ & $\begin{array}{l}3 \text { hours - } \\
5 \text { days }\end{array}$ & $\begin{array}{l}2 \text { hours - } \\
4 \text { days }\end{array}$ \\
\hline first aid courses (distance), range & $\begin{array}{l}25 \min - \\
90 \min \end{array}$ & 3 days & $\begin{array}{l}1 \text { hour - } \\
3 \text { hours }\end{array}$ \\
\hline $\begin{array}{l}\text { BLS-devoted courses (classroom/blended), } \\
\text { range }\end{array}$ & $\begin{array}{l}2 \text { hours - } \\
1 \text { day }\end{array}$ & NS & $\begin{array}{l}1 \text { hour - } \\
6 \text { hours }\end{array}$ \\
\hline BLS-devoted courses (distance), range & $\begin{array}{l}19 \min - \\
2 \text { hours }\end{array}$ & $\begin{array}{l}30 \mathrm{~min}- \\
90 \mathrm{~min}\end{array}$ & NS \\
\hline NS, $n$ (\%) & $13(25)$ & $7(24)$ & $22(11)$ \\
\hline Maximum group size, mean (range) & $25(10-30)$ & $10(9-10)$ & $12(6-15)$ \\
\hline NS, $n$ (\%) & $42(79)$ & $26(90)$ & $153(74)$ \\
\hline \multicolumn{4}{|l|}{ Cost per trainee (US dollar) } \\
\hline $\begin{array}{l}\text { first aid courses (classroom/blended), mean } \\
\text { (range) }\end{array}$ & $85(8-282)$ & $91(36-193)$ & $\begin{array}{l}137(31- \\
905)\end{array}$ \\
\hline first aid courses (distance), mean (range) & $61(35-139)$ & 110 (NA) & $27(19-31)$ \\
\hline $\begin{array}{l}\text { BLS-devoted courses (classroom/blended), } \\
\text { mean (range) }\end{array}$ & $42(28-56)$ & $\begin{array}{l}111(83- \\
138)\end{array}$ & $66(31-157)$ \\
\hline $\begin{array}{l}\text { BLS-devoted courses (distance), mean } \\
\text { (range) }\end{array}$ & $36(10-95)$ & $35(25-50)$ & $24(23-25)$ \\
\hline free classroom/blended courses, $n$ (\%) & $0(0)$ & $0(0)$ & $10(5)$ \\
\hline free distance courses, $n(\%)$ & $7(35)$ & $0(0)$ & $1(0.5)$ \\
\hline NS, $n(\%)$ & $24(45)$ & $6(21)$ & $67(32)$ \\
\hline
\end{tabular}

Notes: * First aid courses containing BLS as part of a syllabus. ${ }^{\dagger}$ Two courses for Nigeria propose various learning methods (classroom/ blended and classroom/distance). Abbreviations: AED - automated external defibrillator; BLS - basic life support; NA - not applicable; NS - not specified.

Conclusions. Compared to the UK, there are very limited opportunities to get trained in BLS for a lay person in India and Nigeria. Nationwide administrative arrangements are urgently needed to increase access of the general public to resuscitation training and improve outcomes in cardiac arrest victims. 